\title{
Improved Vessel Enhancement for Fully Automatic Coronary Modeling
}

\author{
Vincent Auvray $^{a}$, Uwe Jandt ${ }^{b}$, Raoul Florent ${ }^{a}$ and Dirk Schäfer ${ }^{b}$ \\ ${ }^{a}$ Medisys Research Lab, Philips Healthcare, 51 rue Carnot, 92156 Suresnes, France; \\ ${ }^{b}$ Philips Research Europe - Hamburg, Sector Medical Imaging Systems, Roentgenstrasse 24-26, \\ 22335 Hamburg, Germany
}

\begin{abstract}
3D coronary modeling extracts the centerlines and width of the coronary arteries from a rotational sequence of angiographies. This process heavily relies on a preliminary filtering of the $2 \mathrm{D}$ angiograms that enhances the vessels. We propose an improved vessel enhancement method specifically designed for this application. It keeps the advantages of Hessian-based extraction methods (speed, robustness, multiscale) while bypassing its more important limitations: the blurring of bifurcations, and the incomplete filling of very large vessels.

The major contributions of this paper are twofold. First, the classical centered kernel used in Hessian-based methods is substituted with an elongated off-centered kernel. The new filter detects the different orientations involved at a bifurcation: it can answer properly to 'half vessels' beginning at the considered pixel (as opposed to the centered classical filter). The proposed "semi-oriented ridge" filter is also more robust to noise, and it stays multi-scale and quickly computable.

Second, an original bifurcation detection and enhancement method is presented, based on the following heuristics: "bifurcations have three vessels (at least) in their immediate neighborhood". More precisely, the semi-oriented ridges answers in each tested orientation $\theta \in]-\pi, \pi]$ are stored in a circular histogram. The proposed bifurcation energy is the height of the third peak in this histogram: it will have a significant value at bifurcations only.

The performance of the complete framework is demonstrated both on the produced vessel maps and on the final modeling results.
\end{abstract}

\section{INTRODUCTION}

In clinical practice, coronary angiography is mainly limited to the direct analysis of $2 \mathrm{D} \mathrm{X}$-ray projections. This can lead to misinterpretations of the actual vessel structure, caused by vessel foreshortening and overlap. The fully automatic 3D modelling of the coronary artery tree proposed in [1] tackles this problem, based on X-ray rotational angiography.

Automatic modelling heavily depends on its first step: the X-ray projections are initially filtered to enhance vessel-like structures and penalize other objects - yielding a vesselness map (Fig.1). This paper presents an efficient vesselness map computation method specifically designed for that application. It alleviates the two major drawbacks of classical vesselness maps for 3D coronary modelling: the imperfect large vessel filling and the poor performance at bifurcations.

The paper is organized as follows: Section 2 describes the considered automatic 3D modelling method [1], and details the limitations of the current vesselness map. Section 3 proposes a novel vessel enhancement method that is free of the main drawbacks of classical techniques. It partly relies on a bifurcation detection and enhancement module that is detailed in Section 4. Section 5 reports vesselness maps and modelling results, and Section 6 contains concluding remarks.

\section{PROBLEM STATEMENT}

To make this paper self-contained, we briefly review the considered automatic 3D coronary modelling technique based on rotational X-ray angiography [1]. We then focus on the expected vesselness maps properties. 

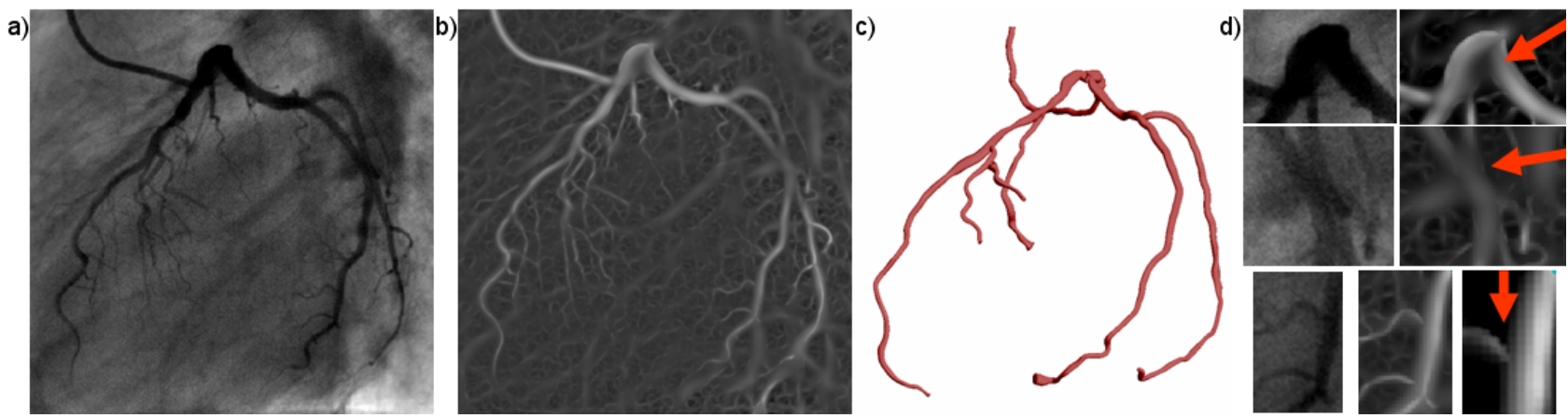

Figure 1. a) original projected angiogram, b) corresponding Hessian-based vessel map, c) targeted modelling (observed from a slightly different perspective) d) details showing the imperfect large vessel filtering and the limited vesselness quality at bifurcation

\subsection{D coronary arteries modelling}

A possible solution to represent the 3D coronary vascular structure is to model it (in its centerlines and vessel width - see Fig.1.c). Such a $3 D$ coronary modelling operation is fast, robust and automatic [1]. It analyses a subset of standard rotational angiography projections belonging to one single cardiac phase. The method is based on a region growing approach, which selects voxels that most probably belong to the vascular structures. More precisely, a measure for the probability $R^{3 D}$ of a voxel $\mathrm{x}^{3 \mathrm{D}}$ to belong to a vessel is calculated:

$$
R^{3 D}\left(\mathbf{x}^{3 \mathbf{D}}\right)=\prod_{n=1}^{N} R_{n}^{2 D}\left(\mathbf{x}^{3 \mathbf{D}}\right)
$$

with $n$ denoting the current projection and $N$ the number of total projections. $R_{n}^{2 D}\left(\mathrm{x}^{3 \mathrm{D}}\right)$ refers to the likelihood that the (cone-beam) forward projection $\mathbf{x}^{2 \mathrm{D}}$ of $\mathbf{x}^{3 \mathrm{D}}$ over the detector plane (at time $n$ ) lies on a coronary artery. $R_{n}^{2 D}$ is computed by previously filtering the projections: this process is the vesselness map computation - or vessel enhancement (Fig.1.b).

The probabilities $R^{3 D}\left(\mathrm{x}^{\mathbf{3}}\right)$ are used to propagate a front that aims at extracting the vessel centerlines. The centerlines representing the most significant vessels are finally selected by a geometry-based weighting criterion.

\subsection{Vessel Map Properties}

Vessel enhancement methods. The whole process therefore relies heavily on the quality of the vessel maps $R_{n}^{2 D}$. They must enhance vessel-like structures and strongly penalize any other objects. Vessel enhancement has been the object of an extensive work (see [2] for a review). Three major categories of approaches can be distinguished: matched filters, Hessian operators and model-based detection methods. Which method to choose is very dependant of the final application.

In our case, two specific requirements have to be considered. The selected method has to be fast since a typical rotational acquisition contains 120 to 260 projections*. Moreover, the chosen vessel enhancement method needs to be multiscale since the considered angiograms can present a variety of vessels sizes (from thin to very large). This militates for Hessian-based vessel enhancement methods.

Hessian-based vessel enhancement methods properties. Tubular objects result in high second order derivatives $D_{2}(., \theta)$ of the image $I$ (orthogonally to the dominant orientation). Hessian-based vessel enhancement methods therefore use:

$$
G\left(\mathbf{x}^{2 \mathbf{D}}\right)=\max _{\theta} D_{2}\left(\mathbf{x}^{2 \mathbf{D}}, \theta\right)
$$

${ }^{*}$ Every one of them must be prefiltered if a modelling is needed in each cardiac phase. 
as a vesselness map $R\left(\mathbf{x}^{\mathbf{2} \mathbf{D}}\right)$ (with $\theta$ the orientation). Now, the second order derivative of the image $I$, in a given direction $\theta$, can be analytically computed [3]:

$$
D_{2}\left(\mathbf{x}^{2 \mathbf{D}}, \theta\right)=-\mathbf{V}_{\theta}^{T} \mathbf{H} \mathbf{V}_{\theta}
$$

with $\mathbf{V}_{\theta}=(\cos \theta, \sin \theta)^{T}$ and $\mathbf{H}$ the Hessian of $I$.

Moreover, it is well known that the maximal value of (3) over every possible orientation can be analytically computed as well (as the Hessian largest eigenvalue). This makes the method particularly fast compared to other vessel enhancement techniques.

Furthermore, Hessian-based vessel enhancement can be used in an efficient multi-scale framework [3]. The maximum local value (3) is simply computed at different resolutions $\sigma^{\dagger}$, yielding different map $R_{\sigma}\left(\mathrm{x}^{2 \mathrm{D}}\right)$. The final vessel map then reads:

$$
R\left(\mathbf{x}^{2 \mathbf{D}}\right)=\max _{\sigma} \sigma^{\gamma} R_{\sigma}\left(\mathbf{x}^{2 \mathbf{D}}\right)
$$

with $\gamma$ a parameter typically in the range $[0.7,2]$. (in practice, we set $\gamma=1.5$ ).

In fact, convolving an image with a Gaussian filter and then looking for its maximum second order derivative boils down to looking for the maximum answers of the image to elongated rotating filters (the so-called ridge filters, pictured on Fig.2) over every possible orientation (defined modulo $\pi$ ). The observation of their impulse response at different resolutions makes clear that they are well-suited to favor elongated objects of different widths.
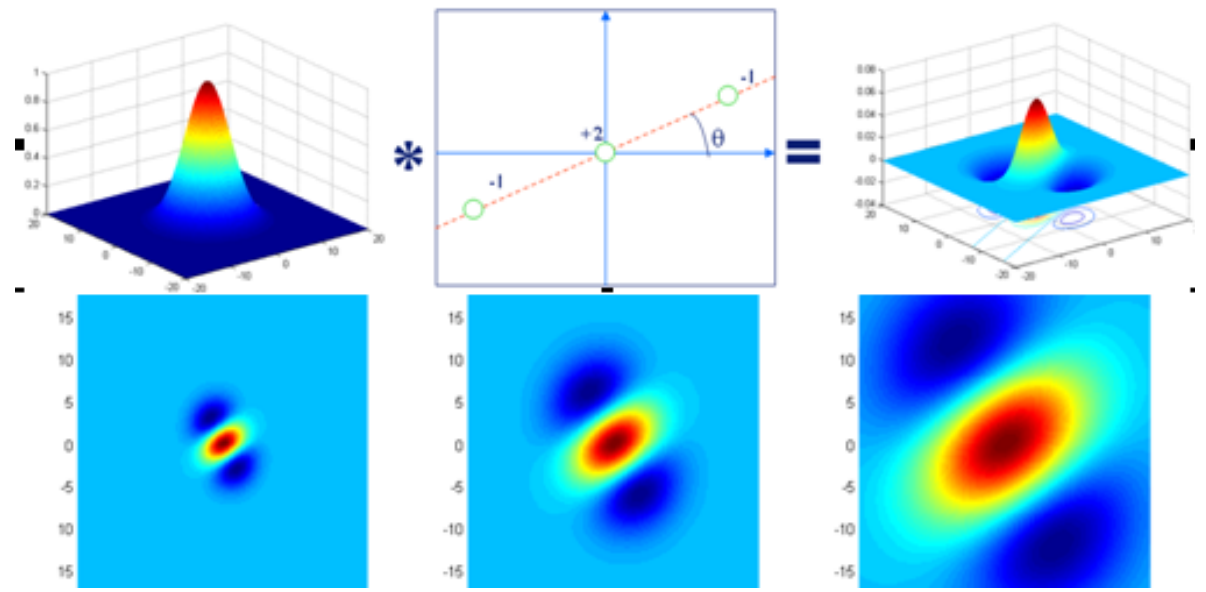

Figure 2. Top: Elongated filter equivalent to the convolution with a Gaussian, followed by a second derivative. Bottom: Elongated filter pictured at different resolutions.

Drawbacks of the Hessian-based vessel enhancement methods. However, every classical vessel enhancement method, including the Hessian-based approach, suffers from the common assumption that there locally exists only one oriented structure [4]. Consequently, they perform poorly at bifurcations points.

This incorrect behavior at bifurcations has also adverse effects at coarse resolutions, yielding a blurring of the thin vessels that are well processed at fine resolutions. As a result, one cannot correctly enhance thin and very large vessels simultaneously. With conventional settings designed to preserve thin vessels, large vessels are not correctly detected: they appear not to be correctly 'filled'.

Both mentioned effects are illustrated on Fig.1.d. They impact negatively the 3D modelling: attenuated bifurcations can stop front propagation, possibly even leading to shortcuts. On the other hand, imperfect large vessel fillings yield an erroneous vascular structure.

\footnotetext{
${ }^{\dagger}$ where $\sigma$ is the standard deviation of the Gaussian filter applied when computing the Hessian matrix
} 


\section{SEMI-ORIENTED RIDGES}

Building on the ridge framework, we propose to construct off-centered, asymmetrical filters able to rotate over a $2 \pi$ angular amplitude (see Fig.3). The major advantage of those new filters is that they are likely to detect the different orientations involved at a bifurcation: they can answer properly to 'half vessels' beginning at the considered pixel (as opposed to the centered classical ridges).

These new filters can be easily computed: they consist in a weighted addition of shifted versions of the classical ridge, along a given direction $\theta$ (in ] $-\pi, \pi]$ ):

$$
S_{\sigma}\left(\mathbf{x}^{2 \mathbf{D}}, \theta\right)=\frac{\sum_{k=0}^{K} w_{k} R_{\sigma}\left(\mathbf{x}^{2 \mathbf{D}}+k \rho \mathbf{V}_{\theta}\right)}{\sum_{k=0}^{K} w_{k}}
$$

However, if used directly this way, semi-oriented ridges would also react in the immediate continuation of elongated objects - even beyond elongated structures. To correct for that effect, the weights $w_{i}$ are associated with adaptive factors:

$$
w_{k}=w_{k}^{\prime} \exp \left(-\frac{\left|I\left(\mathbf{x}^{\mathbf{2}}\right)-I\left(\mathbf{x}^{\mathbf{2 D}}+k \rho \mathbf{V}_{\theta}\right)\right|^{2}}{2 \sigma_{G V}^{2}}\right)
$$

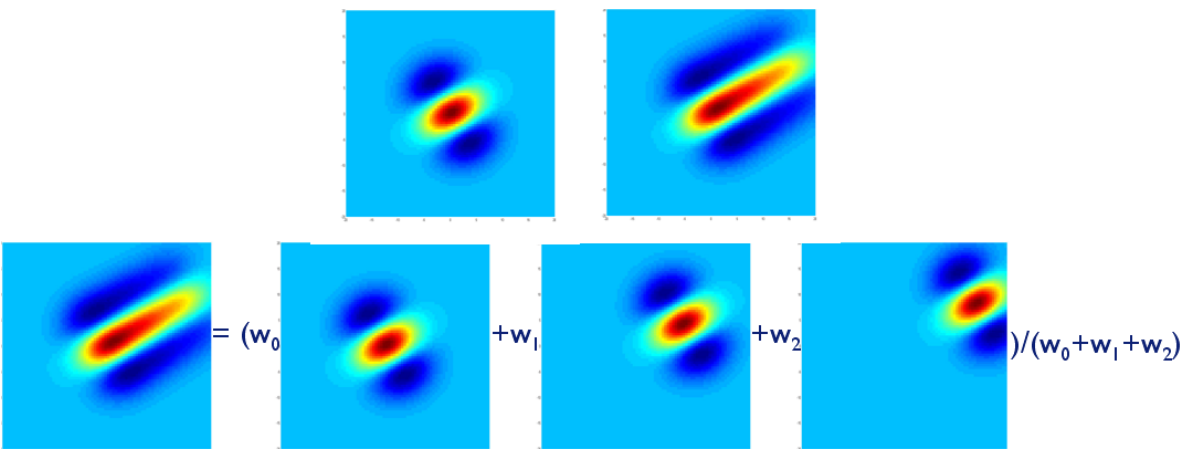

Figure 3. Top: Ridge filter (left) compared to the semi-oriented ridge filter (right). Bottom: Computation of the semioriented filter based on shifted ridge filters.

In practice, $R_{\sigma}\left(\mathbf{x}^{2 \mathrm{D}}, \theta\right)$ is computed following Eq.3, with $K=2$ and $\rho=2 \sigma . \sigma_{G V}$ is set to the image noise level, $w_{0}^{\prime}$ to $1, w_{1}^{\prime}$ to 0.7 and $w_{2}^{\prime}$ to 0.5 . Semi-oriented ridges are used in a multiresolution framework similar to Eq.4.

To summarize, semi-oriented ridges allow to keep the advantages of classical ridges: these filters are well-suited to enhance vessels, they can be computed quickly ${ }^{\ddagger}$ and they are merged in an efficient multiscale framework. Moreover, they better preserve bifurcations, as will be shown in Section 5 .

\section{BIFURCATION ENHANCEMENT}

Even if semi-oriented ridges perform noticeably better than classical ridges at bifurcations, they still tend to blur them at coarse resolution. To preserve bifurcations anyway, we propose to detect and enhance them before coarse resolutions are explored.

\footnotetext{
${ }^{\ddagger}$ Even if less quickly than classical ridges: no analytic formula can be derived to explicitly compute the maximum of $S_{\sigma}(., \theta)$ over every orientations. However, $S_{\sigma}(., \theta)$ can be computed quickly for a given orientation, using Eq.3 and two rigid translations.

The use of the steerable filter theory for the same purpose would require very high order derivatives (given the targeted elongation), and would therefore be much less robust to noise.
} 


\subsection{Bifurcation Energy}

Early works on bifurcation detection [5] exploited local dominant orientations, gathered in an histogram. However, these orientations are defined modulo $\pi$ and therefore hardly suited for bifurcations. Moreover, it is unclear how the considered local neighborhood is selected, though its size is critical. Finally, these methods also tend to classify curved vessels as bifurcations.

Two recent articles proposed to deal with elongated structures including bifurcations without detecting them explicitly. Qian et al proposed to consider a pixel as being part of a vessel if a small circle centered on it crosses at least one vessel [4]. The authors derived from this idea a vessel detection method able to address any local configuration (e.g. bifurcations), but it could be applied in limited situations only (no background). An elegant framework handling mono- and double-orientation detections was proposed in [6], yet it proved to lack robustness when applied in angiography. None of these three approaches is multiscale.

We propose to explicitly characterize bifurcations, based on the following idea: bifurcations have three vessels (at least) in their immediate neighborhood. To study the vessel density in the neighborhood of each pixel $\mathbf{x}^{2 \mathbf{D}}$, we resort to the semi-oriented ridges (Eq.5). Since we are interested in the vessel configuration around $\mathrm{x}^{2 \mathbf{D}}$, we modify the weights, setting $w_{0}$ to $0, w_{1}$ to 0.5 and $w_{2}$ to 1 .

The filter answer in each tested orientation $\theta \in[-\pi, \pi]$ is stored in a circular histogram $(\theta$-addressed). This process is carried out at different scales: every entry in the histogram corresponds to the best filter answer for the considered orientation over the different scales $\sigma$, following Eq.4.

Three kinds of circular histograms can be finally expected. If $\mathbf{x}^{\mathbf{2 D}}$ lies on a linear vessel, the histogram will present two clear peaks. If $\mathbf{x}^{\mathbf{2 D}}$ lies on a bifurcation, the histogram will present at least three peaks, corresponding to the crossed vessels in the neighborhood. Finally, if $\mathbf{x}^{2 \mathrm{D}}$ lies in the background, no clear peaks will appear; only background noise will be present in the histogram.

We therefore choose the height of the third peak of the circular histogram as an indicator for the bifurcation energy $B$. It will have a significant value on bifurcations only. Fig.4 demonstrates the quality of the bifurcation energy $B$ over a typical angiogram.
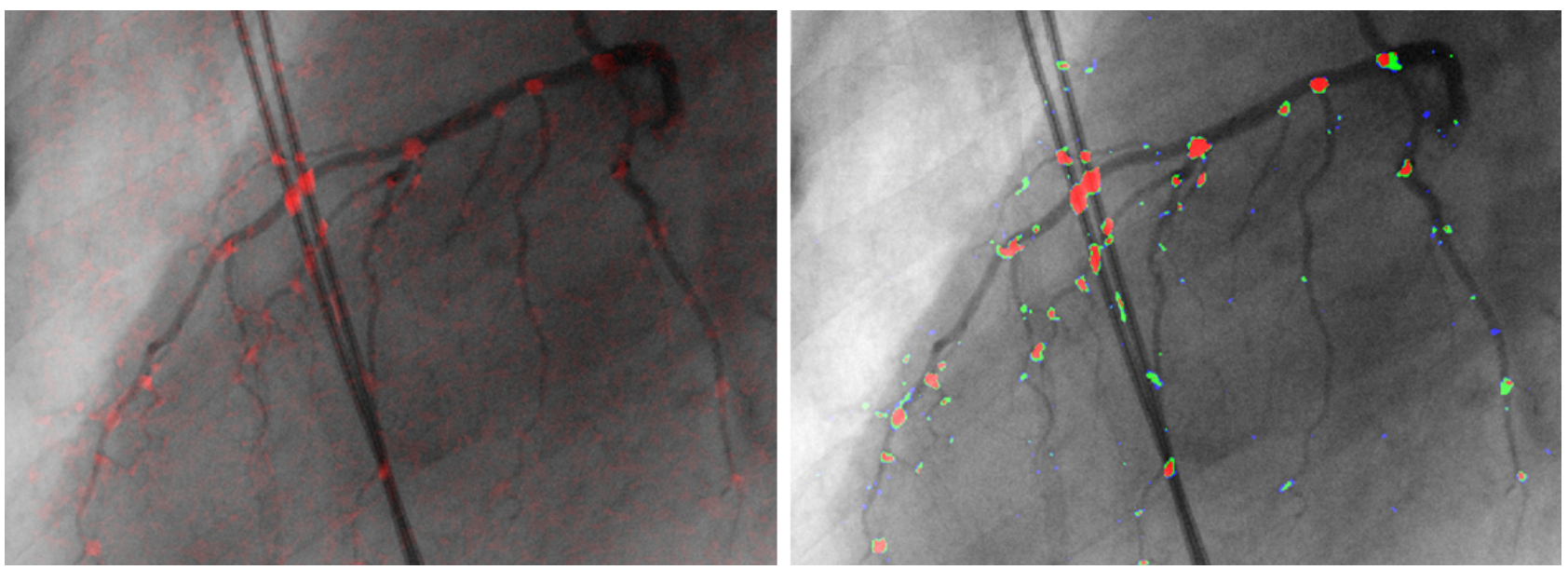

Figure 4. Left: bifurcation energy superimposed to the original image, right: top percentiles of the bifurcation energy (red top $0.5 \%$, green $0.5 \%$ to $1 \%$ and blue 1 to $1.5 \%$ ).

\subsection{Bifurcation Enhancement}

We extract the bifurcation probability $B_{2}$ from the energy $B$ through soft-thresholding. The thresholds are set to ensure that a realistic surface area of the image is labeled as bifurcations - typically 1.5\% (see Fig.4).

Our objective now is to enhance the vesselness where bifurcations $B_{2}$ have been detected. To do so, we resort to a gray-value-based diffusion of the current semi-oriented ridge image $S$. It reads at iteration $i$ :

$$
T^{i}\left(\mathbf{x}^{2 \mathbf{D}}\right)=\frac{\sum_{\mathbf{x}_{2}^{2 \mathbf{D}} \in V\left(\mathbf{x}^{2 \mathbf{D}}\right)} f\left(\left|I\left(\mathbf{x}^{2 \mathbf{D}}\right)-I\left(\mathbf{x}_{\mathbf{2}}^{2 \mathbf{D}}\right)\right|\right) S^{i-1}\left(\mathbf{x}^{2 \mathbf{D}}\right)}{\sum_{\mathbf{x}_{\mathbf{2}}^{2 \mathbf{D}} \in V\left(\mathbf{x}^{2 \mathbf{D}}\right)} f\left(\left|I\left(\mathbf{x}^{2 \mathbf{D}}\right)-I\left(\mathbf{x}_{\mathbf{2}}^{2 \mathbf{D}}\right)\right|\right)}
$$




$$
S^{i}\left(\mathbf{x}^{\mathbf{2} \mathbf{D}}\right)=\left(1-B_{2}\left(\mathbf{x}^{2 \mathbf{D}}\right)\right) S^{i-1}\left(\mathbf{x}^{\mathbf{2} \mathbf{D}}\right)+B_{2}\left(\mathbf{x}^{\mathbf{2} \mathbf{D}}\right) \max \left(S^{i-1}\left(\mathbf{x}^{2 \mathbf{D}}\right), T^{i}\left(\mathbf{x}^{2 \mathbf{D}}\right)\right)
$$

with $S^{i}($.$) the vesselness map at time i$ of the bifurcation enhancement process $\left(S^{0}()=.S().\right), V\left(\mathbf{x}^{2 \mathrm{D}}\right)$ a neighborhood of $\mathbf{x}^{2 \mathbf{D}}$ and $f(t)=\exp \left(-t^{2} /\left(2 \sigma_{G V}\right)^{2}\right)$.

Eq.7 refers to a vesselness diffusion, controlled by the image grayvalues. Vesselness is diffused only within regions having similar grayvalues. Eq.8 integrates the diffused vesselness $T^{i}$ into the vesselness map $S^{i}$ where bifurcations have been detected, only if this increases the local vesselness value .

\section{RESULTS}

The proposed method involves three steps. At fine resolutions (typically $\sigma=2$ to 4 ) the vessel maps are computed using the semi-oriented filters ${ }^{\S}$ (Section 3). Then, bifurcations are detected and enhanced (Section 4 ). And finally, coarse resolutions are explored with semi-oriented ridges $(\sigma=5$ to 7$)$.

We compare on Fig.5 the classical and proposed vessel maps on two examples. Bifurcations are better preserved, and vessels better filled. Moreover, a better vessel to background SNR can be noticed.

Fig.6 presents 3D coronary modelling on two cases, based on the classical and the new proposed vessel maps.
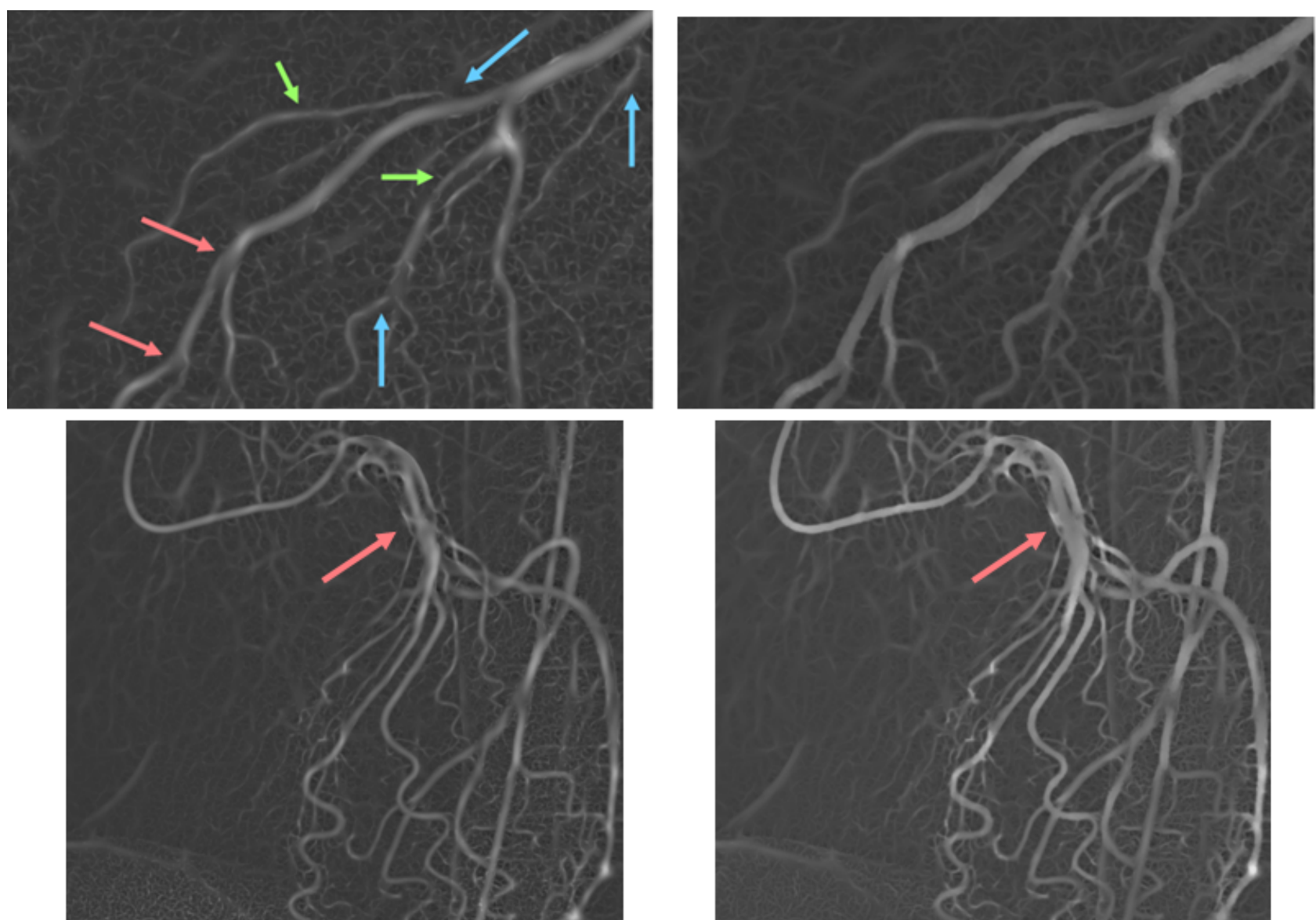

Figure 5. Left: classical ridge vessel map, right: proposed vessel map. Illustration of the better bifurcation enhancement (top), and of the better large vessel filling (bottom).

It shows the extracted centerline reprojected on the vessel maps. The first case shows a better modelling of secondary vessels, and the second example that one shortcut could be avoided.

\footnotetext{
${ }^{\S}$ And the circular histogram values are stored for the subsequent bifurcation detection.
} 

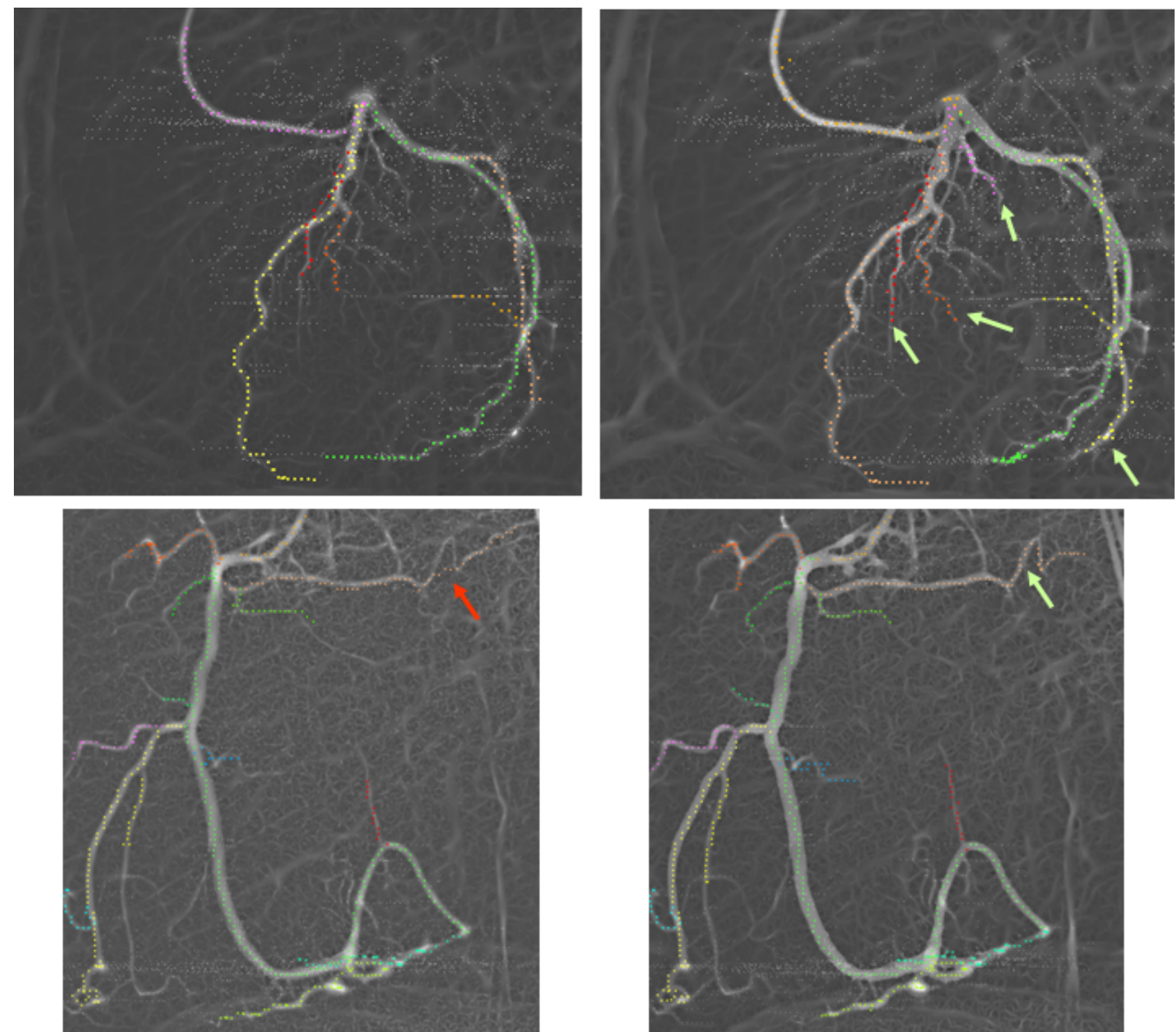

Figure 6. Two modelling examples. The $3 D$ centerlines are reprojected on the vessel maps. Left: classical Hessian-based vessel map, right: proposed vessel map. Top: example showing that thin vessels are better modelled. Bottom: example of an avoided shortcut.

\section{CONCLUSION}

We have presented a novel vessel enhancement method, specifically designed for 3D coronary modelling. Its major contributions are twofold. First, a novel multiscale Hessian-based vessel enhancement method was proposed. It substitutes the classical centered kernel with an elongated off-centered asymmetrical kernel, yielding much better behavior at bifurcations.

Second, an original bifurcation detection and enhancement method is presented. Bifurcations are detected by extracting the third peak of the circular histogram that stores the local vessel density. This can be an interesting output per se.

The performance of the complete framework was demonstrated by visual inspection. Some clear improvements on the final modelling results were also presented. A perspective for the future is to allow for an even larger simultaneous enhancement of thin and very large vessels. 


\section{REFERENCES}

[1] Jandt, U., Schäfer, D., Grass, M., and Rasche, V., "Automatic generation of 3D coronary artery centerlines using rotational X-ray angiography," in [Proc. SPIE Vol. 6510 65104Y-1], (2007).

[2] Kirbas, C. and Quek, F., "A review of vessel extraction techniques and algorithms.," tech. rep., Vision Interfaces and Systems Laboratory (VISLab) (2002).

[3] Lindeberg, T., "Edge detection and ridge detection with automatic scale detection," Int. Journal of Computer Vision 30(2), 117-154 (1998).

[4] Qian, X. et al, "Detection of complex vascular structures using polar neighborhood intensity profile," in [IRRR Comp. Soc. Workshop on Mathematical Methods in Biomedical Image Analysis], 1-8 (2007).

[5] Johanson, B., "Representing multiple orientation in 2D with multiple channel histograms," tech. rep., Comput. Vis. Lab., Linkoeping University (2002).

[6] Aach, T., Mota, C., Stuke, I., Mülich, M., and Barth, E., "Analysis of superimposed oriented patterns," IEEE Transactions on Image Processing 15(12) (2006). 